\title{
Smoking Method of Administration
}

National Cancer Institute

\section{Source}

National Cancer Institute. Smoking Method of Administration. NCI Thesaurus. Code C149363.

The technique of administration of a pharmaceutical product by lighting it in order to evolve smoke that is taken in by the subject. 